\title{
Standard electronic health record (EHR) framework for Indian healthcare system
}

\author{
Manohara M. M. Pai ${ }^{1}$ (D) Raghavendra Ganiga ${ }^{1}$ (D) $\cdot$ Radhika M. Pai $^{1}$ (D) \\ Rajesh Kumar Sinha²
}

Received: 17 August 2020 / Revised: 8 December 2020 / Accepted: 27 December 2020 /

Published online: 27 January 2021

(c) The Author(s) 2021

\begin{abstract}
Digitization of health records in public health facility and its instant availability in the form of electronic records anywhere any time health service is yet to be implemented in developing nations like India and other countries. In India, patient care is mainly delivered through 3 levels namely Primary/Community Healthcare Centre (PHC/CHC), Secondary healthcare centre (District Hospital), and Tertiary Healthcare Centre (National level). The healthcare facilities face many challenges in collecting, processing, and storing these data and managing it without compromising security and privacy. Presently, some of the secondary and tertiary care facilities have started implementing healthcare IT application in terms of Hospital Information System, Hospital Management Information System, Electronic Medical Records (EMR) etc. to manage the patient data in electronic format. However, these systems are developed by different vendors by using different programming languages and databases. This approach makes the system unique but the patient details remains in the same hospital and cannot be shared with other hospitals when patient moves from one hospital to other for advanced or specialized treatment. This is because the data is not interoperable and semantic. In the proposed work, a standard secure Electronic Health Record(EHR) framework is developed using standard medical terminology and coding standards. Implementation of EHR framework for Indian health system will improve the work-flow of health services to the population. EHR at all levels of healthcare system enable efficient and continuous care to the patient.
\end{abstract}

Keywords EHR $\cdot$ Framework $\cdot$ Healthcare $\cdot$ Healthcare Standards $\cdot$ Security

Raghavendra Ganiga

raghavendra.n@manipal.edu

Radhika M. Pai

radhika.pai@manipal.edu

Manohara M. M. Pai

mmm.pai@manipal.edu

1 Department of Information and Communication Technology, Manipal Institute of Technology,

Manipal Academy of Higher Education, Manipal, Karnataka 576104, India

2 Health Information Management-Allied Health Program, Manipal Tata Medical College,

Jamshedpur, Jharkhand 831017, India 


\section{Introduction}

Indian healthcare system follows a diverse system, consisting of a large number of hospitals of various size and units, run by the central and state government (Gopal 2019). In this system, patient care is mainly delivered through Primary/Community Healthcare Centre (PHC/CHC), Secondary Healthcare Centre (District Hospital), and Tertiary Healthcare Centre (National level) (Deodhar 1982; Bagchi 2008). These institutions are functioning under the aegis of the Central and State Government. The private sector institutions, along with the public sector, equally participate in the provision of quality services to the needy population(Chokshi et al. 2016). The different levels of healthcare system is depicted in Fig. 1.

Primary level is the first level of contact of individual or community with the healthcare system. If the patient needs advanced or specialized treatment which is not available at the primary level, he/she is referred to secondary level. Here, the secondary level becomes the first referral level. If the patient further requires the specialized diagnosis and treatment, he/she is then referred to tertiary level. Here, the tertiary level becomes the second referral level.

In India, the healthcare facility is available in both rural and urban areas. Majority of the PHC's are located in the rural areas, where the documentation of care provided to the patient is paper based in the form of reports and records. Large amount of data gets generated every time, when the patient visits the healthcare facility for diagnosis and treatment. The healthcare facilities face many challenges in collecting, processing, and storing these data and managing it without compromising its confidentiality and privacy. Since most of the records are paper based, sometimes, it is difficult for the referred patients to carry their medical reports to every hospital they visit. The paperbased documentation also leads to long queue for the registration and long wait time for the treatment due to unavailability of medical records on time. The manual documentation not only consumes time in capturing but also makes it difficult to share patient data in an instant and real-time manner. Presently some of the healthcare facilities have started using Information Technology(IT) in healthcare to manage patient data in digital format. Even if the health data is in electronic form, it is usually spread across multiple Hospital Information System(HIS), in different data formats and platform. Due to

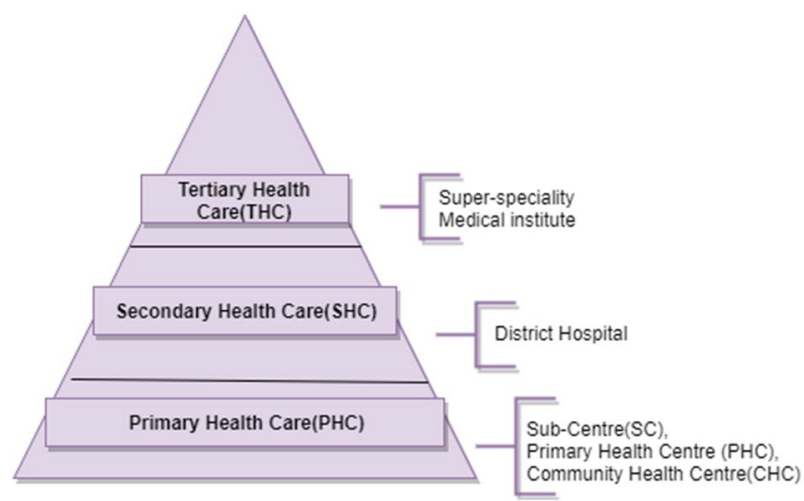

Fig. 1 Levels of healthcare system 
lack of standardization of the records, health information of the patient available in the some healthcare data repository cannot be accessed by the others.

During the last few decades, Information and Communication Technology (I\&CT) has supported the healthcare professionals in managing the information related to patient care, research and education (Sarbadhikari 2018; Balsari et al. 2018). ICTs in healthcare delivery system have great potential to improve the health of the public in both developed and developing countries by enhancing access to health information by all levels of healthcare system. Delivery of patient care is highly dependent on patient health data and the power of ICT mainly lies in its capacity to effectively collect, store, access, analyze and transmit patient health data electronically. ICTs have the potential to significantly contribute in preventive care, improving health service delivery, disease control, health management and research (Courtney et al. 2013).

Healthcare ecosystem consists of doctor, nurse, pharmacist, radiologist, lab technician, and patient. The data generated at the physician's office and other locations of the hospitals such as medical laboratory, medical imaging etc., during clinical encounters are typically managed, stored and maintained by the hospitals for longer period in order to provide patient care and follow-up. Due to large data, hospital sometimes finds it difficult to store and manage health data. Cloud computing has changed the way of managing all levels of healthcare data of the patient and it permits to access data instantly whenever they need by the healthcare service provider (Griebel et al. 2015).

\subsection{Scenario of health information management in Indian hospitals}

Healthcare levels in India follows different approaches to manage the patient health information. Traditionally in India, most of the hospitals manage patient health information in paper based format, including PHC, SHC and THC level. In paper based approach, healthcare providers are unable to provide better care mainly because extremely useful patient health information is unavailable during episode of care. This is mainly due to the unstructured way of keeping the records in paper based system. Presently most of the secondary and tertiary care hospitals have started using computer based record system for managing the health care information (Dick et al. 1997). It allows healthcare professionals to manage medical information in a significant way compared to paper based chart. Healthcare provider can use this technology to store the data such as symptoms, treatment and prescriptions details of each visit of the patient in the hospital data repository. Electronic Medical Record(EMR) is an electronic version of patient health related information gathered from a single healthcare service provider. Hybrid record represents the individual patient health information in both paper based and electronic format. Today, majority of the hospitals maintain and manage health records in the hybrid format (Stausberg et al. 2003; Lærum et al. 2003). Some of the tertiary health centers have moved from the paper-based medical records to the electronic repository, which connects different departments of tertiary health centers to a centralized data warehouse. This electronic repository allows healthcare professionals to access the patient data irrespective of departments. This system assists the healthcare professionals in receiving and sending patient data within the hospital and also controlling the flow of information at the point of care. 


\subsection{Need for electronic health records in Indian hospitals}

Each healthcare organization has a EMR system to capture patient data in an electronic format but complete health information is not available to healthcare professionals during point of care. Siloed vendors use different standards for clinical data exchange. With this approach healthcare user has to use various EMR applications and there is no uniform access. According to Health Information Management Systems Society's (HIMSS) (Sweeney 2017), representation of health information system with a set of silos is as shown in Fig. 2. In a silo system, patient records are spread across different services of the EMR application. If the healthcare professionals would like to access the complete health information about the patient, he/she needs to find the patient records form the various application by logging in multiple times to get the complete details of the patient.

With the Government of India 'Digital Health Mission', there has been a lot of advancements in the conglomerations of Healthcare and IT industry (Srivastava 2016; Sarbadhikari 2019). This movement has led to the development of new methods and tools in maintaining the patient data in the digital form. The Electronic Health Record (EHR) is one such solution to support the healthcare facility, irrespective of levels and sizes to improve patient care by enabling functions that other types of records cannot deliver. The major requirements in healthcare facility is to use interoperability and standardization technique to enable easy sharing and exchange of healthcare data between the various levels. The main foundation for the interoperability is the standard terminology, which improves the effective communication between the two healthcare users (Warren et al. 2015).

Government of India has taken the initiative and formulated and published an EHR standard in September 2013 and consequently revised and published the next version on 31 December 2016 (2016). For EHR standards, Ministry of Health and Family Welfare (MoHFW) suggest the healthcare facility to use the following standards namely

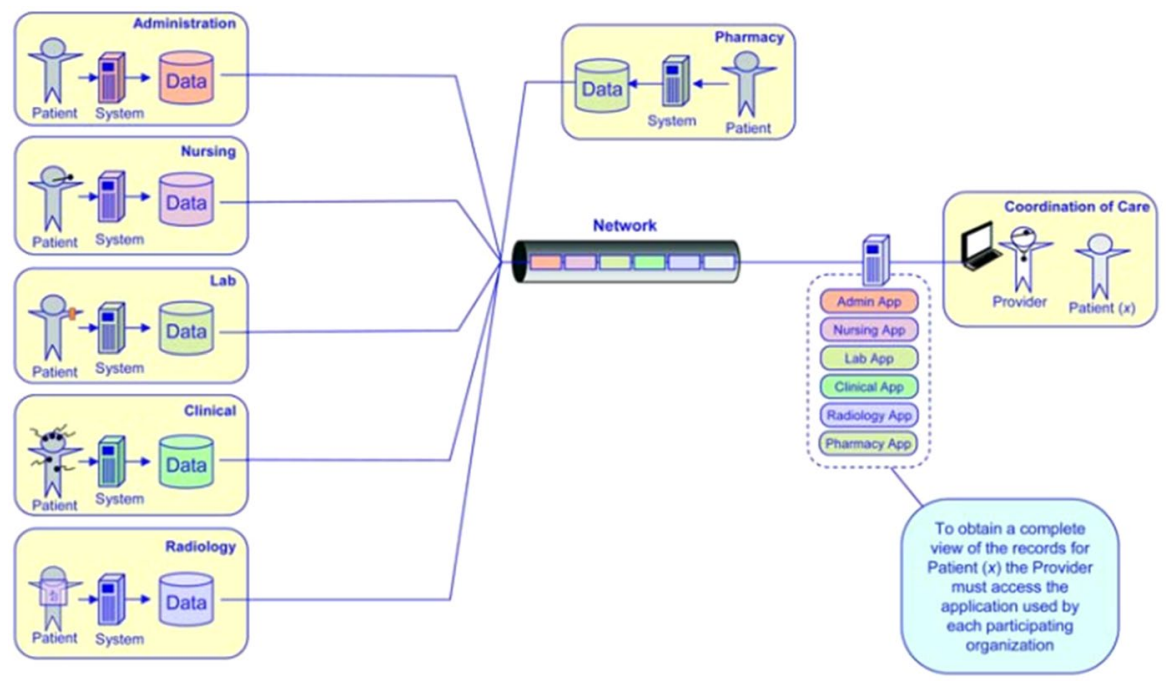

Fig. 2 Health Information System Silos 
Systematized Nomenclature of Medicine - Clinical Terms (SNOMED CT) (2016), International Classification of Diseases (ICD 11) (2016), Logical Observation Identifiers Names and Codes (LOINC) (2016), and National Drug Code(NDC) (2016).

The adoption of terminologies and standard coding system in Health Information System (Sarbadhikari 2019) ensures that data in the system is unambiguous. Content Exchange Standards(CES) are helpful to share clinical information including clinical summaries, prescriptions etc. The Digital Imaging and Communications in Medicine (DICOM) (2016) and Health Level-7(HL-7) (2016) are the CES used for sharing health information with other healthcare levels when patient moves from one healthcare facility to other.

To boost the implementation of digital health in a countrywide manner, the standard committee Ministry of Electronics and Information Technology(Meity)has prepared the draft of National Digital Health Blueprint (NDHB) (2016). The draft was submitted to Ministry of Health and Family Welfare, Government of India on $24^{\text {th }}$ April 2019. Accordingly, NDHB draft was placed for the public domain for comments. The model of the EHR proposed is complying with guidelines outlined in the NDHM document as per EHR minimum dataset requirement (Ganiga et al. 2017, 2020). This EHR will allow the healthcare facility from primary up to tertiary level to capture and share patient data without any restriction and in a secure manner.

\subsection{Health information security and privacy regulations}

The aim of the hospital Health Information System(HIS) is to maintain patient data in a secure manner, considering the privacy rights of the patient and their caregivers. When the records are maintained electronically using EHR, where it has to be shared among multiple healthcare providers at a different level of the healthcare system, chances of the breach in privacy and confidentiality of patient data becomes more, which creates a threat for a healthcare institution (Gkoulalas-Divanis et al. 2014). As the medical records contain sensitive information about the patient, the breach in privacy and confidentiality lead to defamation, discrimination, and stressful conditions to the patient and caregivers. The health record system in India necessarily is safeguarded by the privacy and security standards. Security standards ensures the confidentiality, integrity, and availability of health information.

In addition to the security requirement, EHRs are also regulated by country specific privacy laws. There are few guidelines suggested by United States of America under the Health Insurance Portability and Accountability Act (HIPAA) (Act 1996), 1996 to safeguard the patient health data that are available and maintained using Electronic Health Record. HIPAA defines privacy rules on who has authority to access to health information system and specifies the security measures including administrative, physical and technical safeguard. On December 4, 2019 the Union Cabinet of India accepted the personal data protection bill in the parliament. The purpose of this bill says that sensitive personal data can be processed only with explicit consent of the person and it prevents from the other organization to access without required consent (D. P. A. of India 2019). According to Information Technology Act 2000, data which is stored, transmitted digitally can be protected by the Data Privacy Rules which mainly refers to Sensitive Personal Information (SPI) which is subset of Personal information (PI). The PI includes passwords, financial related information, psychological, physical, mental health condition, biometric, medical record and history of the patient. 
The aim of this research work is to design and develop a cloud based standardized EHR for public healthcare facility which is secure and provides a solution to access patient health information from various level of healthcare system.

This paper is organized as follows. The Literature Survey is described in Sect. 2. Study of the existing health information systems are described in Sect. 3. In Sect. 4 development of standardized Electronic Health Record framework is discussed. Implementation and testing are discussed in Sect. 5. Finally, Sect. 6 concludes the paper.

\section{Literature survey}

The section surveys the various works undertaken in Health Information System(HIS). It also portrays the existing Health Information System in practice with respect to global and national scenario.

\subsection{Health information system - history and evolution}

The history of HIS (Gkoulalas-Divanis et al. 2014; Act 1996; D. P. A. of India 2019; Commission et al. 2015; Affeldt 1980; Wright 2017; Sayles and Gordon 2013; Takeda 1999; Jaroudi and Payne 2019; Hersh 1995) and the evolution of the EHR is shown in Fig. 3. Medical records are an essential feature of any Hospital Management System (HMS) and it has evolved during the past several years. The latest innovations in healthcare Information Technology has changed the way of recording health information. In 1920s (Commission et al. 2015), all medical records were maintained and managed manually with the paper based documentation. Patient visit details, history, diagnosis, lab result, medication and all details were written on the paper and manually managed in the medical record room.

The medical record contained key clinical information about the patient and his care. An accrediting body, Joint Commission on Accreditation of Hospitals (Affeldt 1980) started surveying hospitals and other care facility on regular basis to check the quality of the medical care by using medical record as a tool. With these initiative greatest improvement started in the hospital for standardizing the medical record section with the defined regulations. With this goal in mind, the American College of Surgeons (ACS) (Wright 2017) initiated the use of standards for the hospitals in United States and Canada for enhancing the clinical care setting. The association of professionals exists today under the name of American Health Information Management Association (AHIMA) (Sayles and Gordon 2013).

Medical records are documented in several ways and one such concept used for documentation of the patient health information is Problem Oriented Medical Record (POMR) (Takeda 1999), which mainly focuses on specific problems that patients have. POMR was introduced by Dr. Lawrence Weed in the 1950s which represents most strategic approach to document the patient care.

The SOAP (Subjective, Objective, Assessment, and Plan)is a technique used to capture the patient data in POMR. SOAP improves the patient care and helps healthcare professionals to provide structured care and treatment. It clearly shows what is happening to the patient in an organized way (Jaroudi and Payne 2019).

Advancement in the technology such as the use of computer system in healthcare sector has led to organized way of recording the medical records electronically. Electronic method of storing health information is beneficial to the organization in the technology advancement period. With increased usage of the computers in the hospitals, individual department 

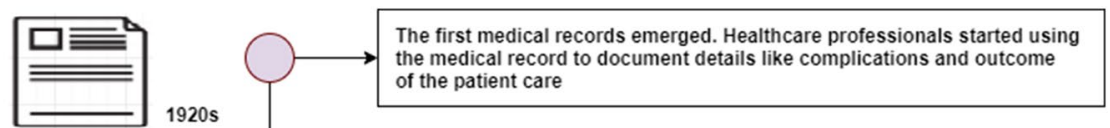

Paper Based medical record

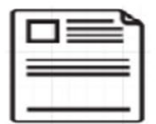

$1928 \mathrm{~s}$

Paper Based medical record

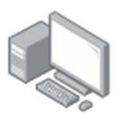

$1960 \mathrm{~s}$

Electronic Medical Record(EMR)

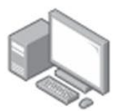

1965

Electronic Medical Record(EMR)

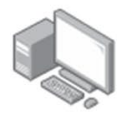

1970s

to

Electronic Medical Record(EMR)

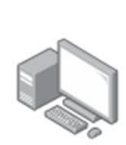

1980 s

to
1990 s

$1990 \mathrm{~s}$

Electronic Health Record(EMR)

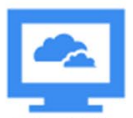

2004

Electronic Health Record(EHR initiative

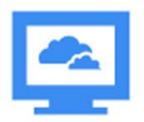

Electronic Health Record(EHR) initiative the medical record to document details like complications and outcome
The American College of Surgeons(ACOS) carried primary responsibility for the establishment of standards for the hospitals of the United State and Canada. Now it is known as the American Health Information Management Association(AHIMA)
Computers meant big mainframes and only the largest providers could dedicate the resources to leverage technology for medical recordkeeping.

When technological innovations such as the use of computers led to the beginning of new approaches to HIM with standardization and storing of medical records. Dr. Lawrence Weed created the first problem-oriented medical record (POMR) to organize the information used in medical records.

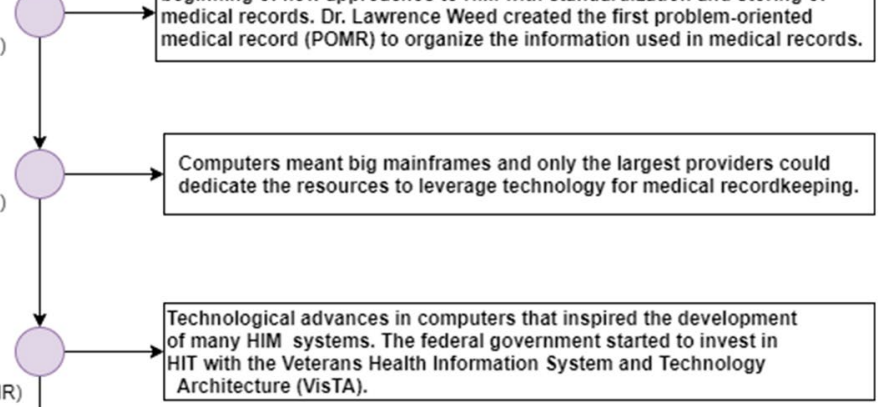

IIT with the Veterans Health Information System and Technology

Architecture (VisTA).

President Barack Obama signed the Health Information Technology for Economic and Clinical Health (HITECH) Act as part of the American Recovery and Reinvestment Act (ARRA) to adopt electronic health records for meaningful use in the hospital. In January 2009, in a speech at Geoge Mansoon University, President Obama said" EHRs will cut waste, eliminate red tape, and reduce the need to repeat expensive medical tests. It will save lives by reducing the deadly but preventable medical errors that pervade our health care system"

In January 2004, President George W. Bush launched an initiative for the widespread adoption of EHRs within the next 10 years. In-state of union address said" By computerizing health records, we can avoid dangerous medical mistakes, reduce costs, and improve care.

Fig. 3 History of HIS and evolution of EHR

in the healthcare organization started using legacy Health Information System(HIS). One such system is EMR (Hersh 1995) which allows transfer of health related data between the healthcare service providers of the same organization and with which healthcare professionals get quickly and easily patient data at their fingertips (Giaedi 2008).

The major changes from EMR to EHR occur in the year 2009 during President Barack Obama term and it is named as the Health Information Technology for Economic and 
Clinical Health Act (HITECH Act) (Gold and McLAUGHLIN 2016). The act is a part of the American Recovery and Reinvestment Act of 2009 (ARRA). The prime focus of HITECH act is to motivate the implementation of the Electronic Health Records and other supporting health IT in the United Sates, so that patient can get better care (Aocnp 2015).

\subsection{Health information system - international scenario}

The literature search evidenced the initiative taken by different countries and their implementation has become a great success in terms of improving the healthcare services by minimizing the errors in documentation and patient care. In 2001, Canada initiated to modernize its Information and Communication Technology (I\&CT) infrastructure in healthcare sector by setting up objectives of standardized procurement practices, sustainable investment, and accelerate innovation adoption scale across the Canadian digital health marketplace (Banff 2015; Rozenblum et al. 2011). In 2015, $91 \%$ of Canadians, and 91,000 clinicians were using the EHR system in their work place for recording health data. The EHR has benefited them in the exchange of clinical information, minimizing the errors, and improving the patient safety and overall quality of healthcare.

England initiated to modernize its healthcare system by launching the national initiative called National Plan for IT (Basu 2017; P. O. of Science and Technology (2016) to provide connectivity between different levels of health records across primary, secondary, and social care by 2020 . With this initiative, summary of health records were created for 54 million persons, i.e. $96 \%$ of the England population. Using this system, healthcare professionals can monitor the summary view of the patient during episode of care.

About $90 \%$ of the private practice physicians in Germany are using EHR system (Lehr 2016). With the modernization of the healthcare system, the patient data safety has been considered as the first priority by the healthcare provider during the point of care. For this specific purpose, secure health network setup is created by Federal Ministry of health Germany for transmitting sensitive medical data over the private link. In case, if the patient health information needs to be shared with other healthcare levels during that time it needs to be connected with the public network. In public network, patient can decide to hide or block any entry in their health record. But during emergency situation, the doctors can get direct access to the patient data using the secret pin provided by Federal Ministry of Health (Rehmann 2016). The EHR system in private sector focuses to improve the quality of services with reduced cost, and enables better data for health care management.

To modernize the healthcare system in Korea, nationwide (Lee et al. 2015) Health Information Exchange (HIE) platform is being built. With this initiation, most of the tertiary hospitals have started using EHRs to exchange among the healthcare facilities. The platform is connected with Application Program Interfaces (APIs) to add different services, include document registry and repository, and a master patient index.

Bahga and Madisetti (2013) discussed about Veterans Health Information Systems and Technology Architecture (VistA) which is the traditional health record system used in United States and covers roughly $25 \%$ of the nation's population. VistA is a collection of 168 application packages /modules. It is not a single application. They also discussed about the limitations of (VistA) with respect to the Design methodology, data interoperability, scalability and performance. Traditional health systems use the unstructured way of recording patient data, and each clinical concept is maintained as a separate table which leads to a large number of tables in the database. Also, various forms of data stored in generic structure method leads to the problem of interoperability when sharing with the other hospitals. 
They also discussed that the using different technical and semantic standards has resulted in conflicts in traditional EHR which ultimately lead to the problem of data integration and interoperability. Different healthcare standards, different technology, and different languages also lead to the problem of exchange of data between two healthcare systems. To overcome this they have proposed two cloud based models for the United States hospital to integrate different health services together namely Open Vista and Cloud Health Information Systems Technology Architecture (CHISTAR).

\subsection{Health information system - national scenario}

Indian Healthcare System has also evidenced the development of ICT application but mainly limited to only few private hospitals such as Max Health (Soi 2019), Apollo (J. C. International 2019), Sankara Nethralaya (M. R. Foundation 2019), Fortis, etc. All India Institute of Medical Sciences (AIIMS) and the Postgraduate Institute of Medical Education and Research (PGIMER) are major public hospitals which have Electronic Medical Record (EMR) in place to share the patient data with other departments of the same group of hospitals (Sharma 2018). The implementation covers significant aspects from registration and billing to laboratory and clinical data. In all the hospitals, the developed EMR are rarely exchanged between the same group of hospitals. When the patient is referred to the other hospital, the patient data remains in the same hospital and patient gets the print copy of the records for further consultation in hospitals, where they are referred. The need of the hour would be to implement the EHR and integrate all the levels of the public health system, where the movement of patient data becomes easier and safer during and after the point of care.

\section{Study of the eexisting healthcare information system}

To design and develop a new IT application requires assessment of the utilization pattern of the existing system among the end users. The data on the assessment helps in understanding the end users satisfaction level with the existing system and assist the planner in planning of better system (Kruse et al. 2018). The public healthcare facility has been visited and the questionnaires were distributed to the healthcare professionals and interviews conducted to understand the significance of digital based system at all levels of healthcare facility. The permission was taken from the District Health Officer (DHO) to visit the various healthcare centers. Following are the government primary healthcare centers visited Sub Center: Moodbettu, Udupi,India, Primary Health Center: Guddeangadi-Padukone, India and Community Health Center: Byndoor, India during December 2016 to February 2017. Firstly, the respondents were briefed with the research and its objectives and the reason of the study. Based on the questionnaire the issues have been recorded and considered into the research to build the EHR system.

The Table 1 presents the result of the survey conducted among the public health care facility to know about the present Health Information System. The result of the study indicated that Primary levels healthcare such as Sub Center (SC), Primary Healthcare Center (PHC) and Community Health Centers (CHC) are managing patient health information in the paper based record. For managing laboratory result and medical imaging they are using hybrid record which is a mixture of both paper based and digital. 
Table 1 Distribution and use of information storage methods in healthcare system

\begin{tabular}{llll}
\hline $\begin{array}{l}\text { Patient Health Information Manage- } \\
\text { ment }\end{array}$ & Paper Based & $\begin{array}{l}\text { Hybrid Record } \\
\text { (Paper+Digital) }\end{array}$ & Fully Digital \\
\hline Demographic Details & $\checkmark$ & $x$ & $x$ \\
Physician and nurse notes & $\checkmark$ & $x$ & $x$ \\
Patient history & $\checkmark$ & $x$ & $x$ \\
Clinical exam observation & $\checkmark$ & $x$ & $x$ \\
Diagnosis details & $\checkmark$ & $x$ & $x$ \\
Treatment procedure & $\checkmark$ & $x$ & $x$ \\
Lab test details & $\checkmark$ & $\checkmark$ & $x$ \\
Medication details & $\checkmark$ & $x$ & $x$ \\
Medical Images & $\checkmark$ & $\checkmark$ & $x$ \\
\hline
\end{tabular}

During public health level visit it is observed that when patient visits the hospital, a token number is issued. The patient must wait till their turn comes and later healthcare professionals records vital sign and diagnosis details of the patient in a case sheet book. During the next visit to the hospital the patients are required to bring the case sheet book for updating the health information. The physician updates the case sheet during every visit with the following information including diagnosis details, investigation result, medications details etc. In PHCs patient's case sheet is the only the record to give the details about the health information. The case sheet is the paper based physical copy and it is vulnerable to damage and over time reduce its quality. This is the only one way of portability solution if the patient moves to the different healthcare level.

Medical documentation at the PHC level is mainly paper based and they maintain registration book, examination book, and the treatment book. The five year old active patient records are maintained in the front desk of the Medical Record Department (MRD) for faster access. All other records are maintained in the repository which are still available for future reference. Healthcare facility staff needs to spend enormous amount of time and effort during the point of care and it becomes a cumbersome process.

During the next visit to PHCs, the IT-infrastructure facility used for managing and maintaining the patient information was assessed and is depicted in Table 2. It shows the present infrastructure facility in PHC levels.

To implement IT application infrastructure in healthcare system especially in rural places, the main obstacle is requirement of the high speed Internet connectivity. To address this national level problem, the Government of India has proposed a new project BharatNet for providing high-speed broadband optical connectivity to all rural areas. With this communication infrastructure all rural places in India will get 1 Gbps (Gigabyte per

Table 2 Infrastructure facility in PHC levels

\begin{tabular}{lllll}
\hline Levels and Facilty & $\begin{array}{l}\text { Total computer } \\
\text { facility }\end{array}$ & $\begin{array}{l}\text { Router facility } \\
\text { (Broadband) }\end{array}$ & Printer & Scanner \\
\hline Sub Centre(SC) & 0 & 0 & 0 & 0 \\
Primary Health Centre(PHC) & 1 & 1 & 1 & 1 \\
Community Health Centre (CHC) & 2 & 1 & 2 & 2 \\
\hline
\end{tabular}


second) bandwidth capacity at rural level setting. Additionally, the government requested Telecommunications(Telecom) company to list all unconnected villages in India and have planned to connect with Telecom services by 2020 .

To understand the way of managing the health record at Secondary and Tertiary level, District hospital, Udupi and Kasturba Hospital, Manipal were visited. During the healthcare visit, it is observed that recording patient health information is done with the hybrid record structure which is a combination of both paper and electronic record. Most of the healthcare facility manages the medical records in paper and few levels maintain in digital form. These include registration information, administrative, and billing data. Many of the documents included in a paper based record consists of demographic data, vital signs, patient health histories, diagnosis details, medications, allergies, lab test result and medical imaging etc. As technology advanced, few healthcare facilities in India have started implementing Electronic Medical Record (EMR). Often these records are not integrated with other healthcare facility and they are recorded and stored in the HIS system which is developed using proprietary software. When the patient is referred to the other hospital, the patient data remains in the same hospital.

During assessment of the existing system in practice, it was observed clearly that the system partially assists them in documenting the patient information, patient care, and reporting but does not support sharing of health information between the different levels of healthcare facility. Most of the respondents felt that the development of the Electronic Health Records(EHR) application reduces the hospital cost in the long run. For healthcare staff, it reduces their workload in terms of documentation and reporting. It also increases the productivity of the various levels of healthcare facility. The respondent expected that the proposed system should support them in clinical decision making through the instant access of the clinical standards, practice guidelines and patient information. They also commented that the healthcare application should be user friendly. It should have the features for the easy documentation, report generation and also assist in improving the clinical outcome of the patient.

Considering the rural and urban healthcare system, the way of managing the patient health information in mind, development of standardized EHR has been proposed with the objectives to reduce healthcare professionals time in information management and retrieval, and enhance their productivity and performance by assisting them with rich source of data in standard coding system. The need of the hour would be to implement the EHR and integrate various levels of public health facility, where the movement of patient data becomes easier and safer during and after the point of care.

\section{Development of standardized electronic health record (EHR)}

Electronic way of recording patient health information has grown progressively in recent years. The patient data present in digital format is stored in the individual machine and individual care delivery setting. These distinct island of information could hinder critical sharing of data between the various levels of healthcare facility. Standards play a major role in improving the interoperability of HIS. Thus standard coding provides easy sharing of health information, reduces uncertainty, improves work flow and accurate analysis of healthcare data.

The primary aim of the healthcare information system is to deliver lifelong clinical care at all times. To meet this requirement, syntactic and semantic interoperability of 
data should be maintained at all times. In a country like India, with its large population spread across a large geographical landscape, to make the patient data accessible by all levels of healthcare providers is difficult as the data is not interoperable and semantic. To meet consistent representation of the interoperability requirements, standardizing vocabularies, or mapping between different vocabularies are necessary. Including standard terminologies and codes in computer processable presentation into a HIS support automation and ensure that data is in an analyzable state at all times. The different standards incorporated in the developed system are depicted in the Fig. 4.

With the EHR, it is possible to capture and store distinct patient health data and be made accessible at point of care including PHC, SHC and THC. EHR contains health information about patient history, laboratory test reports, and diagnosis images stored in a digital format which is available to healthcare providers through computer network (Ministry of health and family welfare 2013). Compared to EMR, the EHR has a more comprehensive report of the patients overall health. The record may be significant or not depending on the current problem that patient is suffering from. Thus, the EHR record provides the information of various clinical encounters and represents in chronological order to support healthcare professionals in providing quality healthcare.

Cloud computing (Guo et al. 2012) has emerged as a main role in providing healthcare IT solution. Therefore, healthcare organizations can largely benefit from cloud infrastructure which could be an excellent solution for the country's needs to have improved healthcare in all levels of healthcare system.

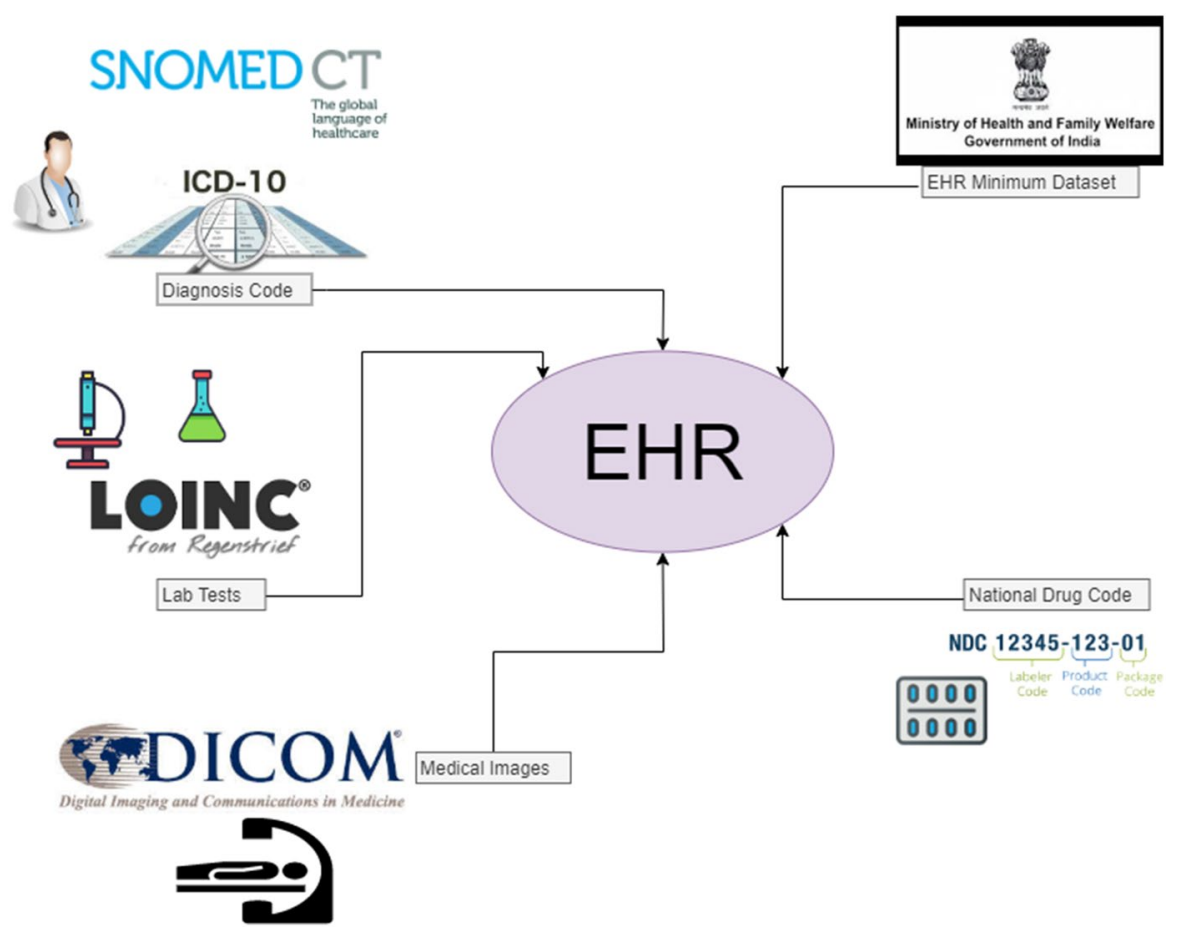

Fig. 4 Electronic Health Record Standards 
The proposed EHR model connects all the users of health information system into one single point to record and share the patient health information through EHR network. The primary purpose is to enable efficient and continuous care of the patient. It is a cloud based framework which enables all levels of healthcare professionals to contribute to the EHR content. In this cloud resources are shared with different healthcare sector such as primary, secondary and tertiary care levels. All the data captured from each healthcare setting is processed and stored in global cloud data base called EHR universal health service provider database. During point of care, healthcare professionals can access patient data securely with EHR web application for the coordination of the care. To develop an integrated system, there is a need to collect the health data from the various modules including administrative, physician's, nursing, laboratories, radiology, pharmacy etc. The different components of the EHR system is depicted in Fig. 5.

The first component of the EHR system is the Administrative system module, where data such as patient registration details (Patient demographic data), admission, discharge, and transfer of record from one hospital to other is maintained. The second component of the system is nursing module, where mainly apparent information of the patient is recorded such as height, weight, blood pressure and BMI values. The third component of EHR includes laboratory data such as microbiology, biochemistry etc., which plays an essential part in clinical process by furnishing relevant data to healthcare service provider. Approximately $60 \%$ to $70 \%$ of the quality decisions are decided based on the lab results. The fourth module includes radiology related data and image such as X-ray, CT Scan, MRI. Medical imaging system called Picture Archiving Communication System (PACS), is an imaging technology for managing digital images. The important and key component of EHR system is clinical documentation module to capture

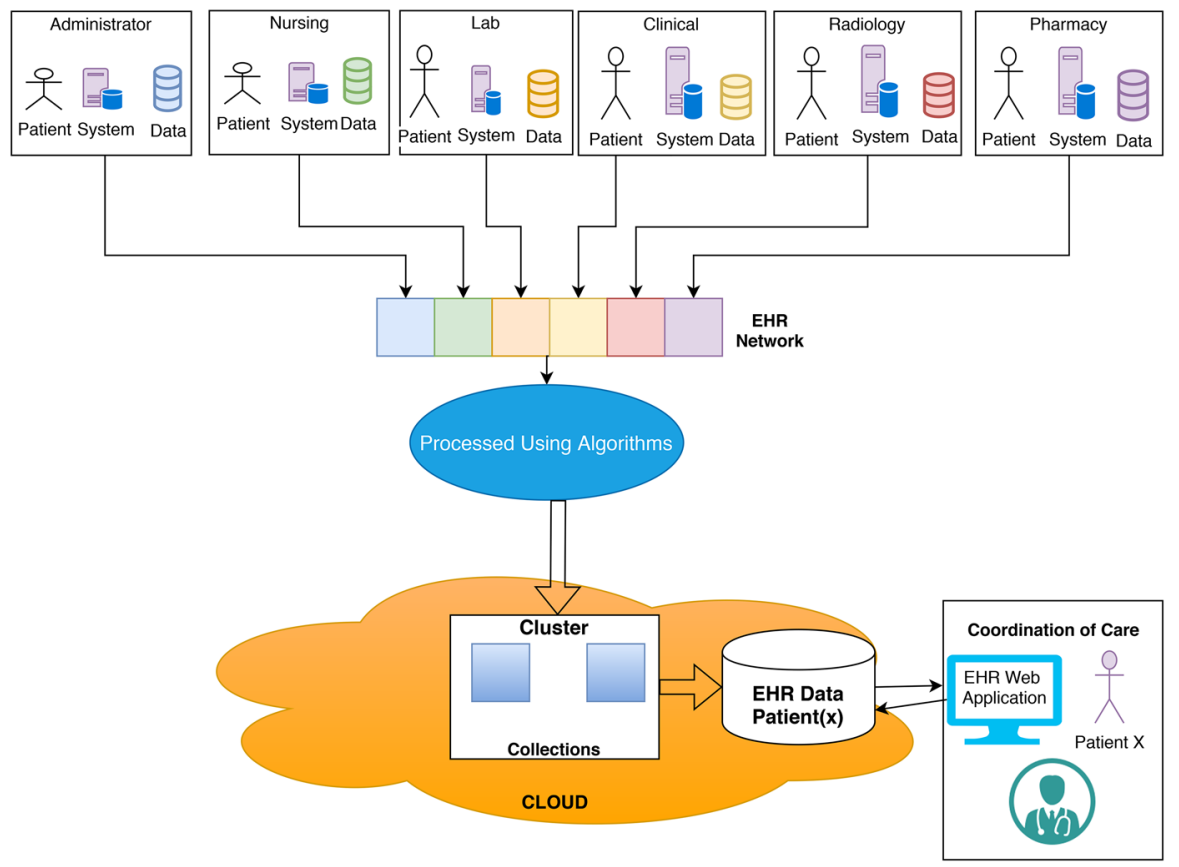

Fig. 5 Proposed EHR Model for the coordination of the care 
patient's clinical data such as diagnosis, procedure, complication and medication. A clinical document in the electronic form helps the healthcare provider to validate and to provide quality care to the patient. The EHR serve the purposes by providing accurate, complete, concise information of the patient, which helps next health care service provider to take quality decision. Another important module in the EHR system is pharmacy, which holds the comprehensive medication history including medication name, drug code, drug name, dosage, quantity, and allergic reaction etc, which results in safe and effective medication to the patient.

The proposed model uses the ID of the Aadhaar or any other valid document the patient is carrying during the first visit to the hospital. This number is used to generate unique ID of the patient. Once the National Digital Health Mission (NDHM) is implemented all over the state, same ID can be used in place of the unique ID generated in our system to access the patient details. The developed model uses MongoDB Atlas Cloud Cluster which store the varieties of health data and it has a multiple collections to form a cluster in the cloud. For retrieval operations, information extracted from the collections in the cluster are processed and then displayed to the end-user. For storing, data which is generated from user inputs, is retrieved from the web application and processed to attain certain standard formats before storing in the collections. In this way a systematic data flow is established and sanctity of the model is maintained. Web based EHR system that is developed is platform independent application which runs on any computer, laptop, mobile phone which is connected to the Internet. An EHR application generally performs two major functions Read and Write. For Read operations, information is extracted from the specific collections in the cluster then processed using algorithms mentioned ahead and then displayed to the end-user. While, for Write operation, information which is generated from user inputs, is retrieved from the website and processed to attain certain standard formats before storing in the collections. In this manner, a systematic data flow is established and sanctity of the model is maintained. Cloud storage helps our work to attain a global platform which is accessible from every part of the world independent of time and date. These few factors guide the model in attaining EHR fluidity by making patient records globally available to verified users.

\subsection{EHR system design}

In software development life cycle, design is the most essential phase. System designer needs to carryout careful planning and thinking which helps to understand how various parts of the system are going to achieve the desired goal. Design should be done with extreme care because during this stage if it has any errors, then that will affect the overall performance of the system. During requirement analysis phase, requirements are collected and specified in the document. Design is the second phase where it can provide a solution to the problem. This phase contains set of iterative steps which enables the designer to define all features of the system to be built.

Design basically acts as a bridge between system requirement specification and final solution for satisfying the requirement (Campbell 2004). The design of a system is essentially a blue print or a plan for the solution of the problem by the system. The content required for developing EHR system is classified into various sections meant to capture vital aspects of a patient's health and medical status. The activity diagram of the EHR system is depicted in Fig. 6. 


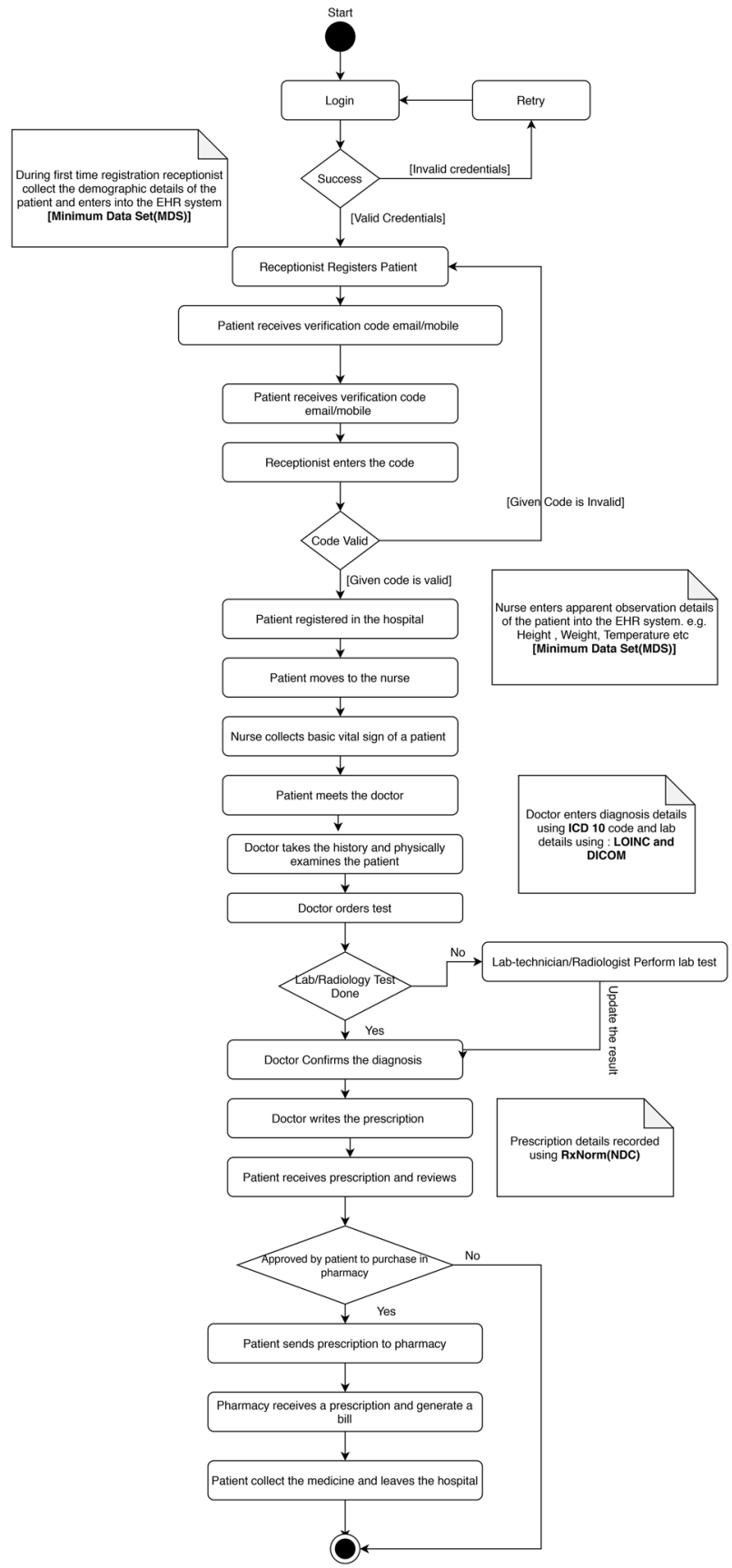

Fig. 6 Overall activity work-flow of EHR System 


\subsubsection{Patient demographics}

One of the main responsibility of the healthcare professionals is to collect the demographic details of the patient accurately when a patient visits the hospital for the first time. Collecting demographic details in consistent manner help the hospital staff to address the unique needs of the patient. The patient demographic section includes details such as name, address, email, contact number, gender, marital status, occupation, emergency contact, location, previous diagnosis etc. EHR also has a support of including medical insurance and healthcare financing details.

\subsubsection{Patient health history and medications}

The EHR system allows healthcare professionals to capture the patient's past medical history and present symptoms. Details of past illness such as hypertension, diabetics, kidney disease, heart disease, asthma, tuberculosis and other needs to be recoded for better diagnosis of the current illness. The various lab tests and multimedia images need to be present to the doctor along with the EHR contents. The drug history mainly captures the list of drugs that the patient was taking prior to the admission in the hospital. In addition to this, EHR system has provision to provide details of drug allergies, dosage, duration, recently stopped medicine. Addiction history such as smoking, tobacco, and alcohol details need to be recorded. The health history of the patient and the patient's family helps to address acute health problems and minimize the chronic condition of the patient.

\subsubsection{Patient clinical encounters}

Clinical encounter provides a principal focus to provide patient centric medical care. During clinical encounter healthcare professionals can get quality data to treat the patient better. Details for the clinical encounter are captured in different healthcare levels and the details are displayed when patient visits the hospital for the current treatment. Details includes physical examination, observations, diagnosis, lab investigation, encounter(interaction between the patient and doctors) type, demographic details and patient history.

\subsubsection{Security framework}

Patient and healthcare professionals are the primary owners of the EHR. It is a confidential communication between patient and healthcare staff and it cannot be released without the permission. A health record is the most important record for healthcare organization. All reports i.e. lab records, X-ray, ultrasound, Magnetic Resonance Imaging (MRI), Computed Tomography (CT-scan), and prescription details should be signed by a doctor before issuing the document or transferring the record from one healthcare levels to others.

Health Information System contains sensitive information about the patient and the data needs to be protected from the unauthorized users, to safeguard the information. To enable sharing the data among multiple healthcare providers in a secure manner a secure EHR framework is developed. The various attacks and vulnerabilities have been identified on the developed EHR model. Potential threats are identified in the EHR system by using STRIDE model and the amount of risk posed to the systems is calculated using DREAD model (Ganiga et al. 2020). A new framework for highly secure sharing of EHR 
in cloud environment is developed which mainly focuses on Authentication, Authorization and Attribute based encryption. SAML-SSO authentication model is adopted in EHR to access multiple services of health information after signing in only once which improves the security and increases the access speed of the health information service. For Authorization Oauth2.0 is used which gives resource access in the cloud based environment. ABE eases the process of managing key for the encryption and it makes it possible to access the patient health information based on the professional roles, experience, and specialization of the doctor and health-care levels. Various modules for EHR has been developed for Indian health-care scenario. Identified security features of EHR ensures a lowest risk of breach and enhanced control over the system.

\section{Implementation and validation}

Healthcare IT sector in India is expected to reach $\$ 280.25$ billion by 2021 , with an annual growth rate of $15.9 \%$ (Outlook 2019). Especially EHR system needs to satisfy quality requirements to provide better care to the patient. To achieve this software testing is necessary for every healthcare industry. The testing is needed for EHR system to validate the functionalities, interoperability, compliance and performance. During the software development life cycle, application developer should give suitable time and testing effort for the developing system. Web application consists of numerous application entries and exit paths, different type of browser with end users, people with varying technical skills using software which makes testing application a challenging task. However in this software each module is tested individually with some input. All the requirements were preserved and taken care while testing each module individually (N. H. I. Technology 2016). There are noteworthy potential benefits of the EHR systems are as given below:

\subsection{Expedited registration}

About 100 patient records are entered into the EHR system with the help of healthcare professionals. It was observed that it allows easy way of recording patient record as compared to traditional system. Patients had to be registered only once and it was a matter of few minutes. In earlier system patients had to visit the hospital and manually fill long and tedious forms before being officially registered in the hospital and then the administration had to maintain all these records on paper. The proposed model overcame this problem and patient registration was made much more efficient and less cumbersome for the administration.

\subsection{Digital signature}

Digital signature are the public key primitives of message authentication which uses two mutually authenticating cryptographic keys. A digital signature is one of the main aspect of ensuring security and integrity of the patient data (Campbell 2004). In healthcare system, medical forms and prescription details of the patient needs to be signed digitally to prevent from the unauthorized access, tampering and forging a signature. Doctor from PHC can sign a document using digital signature procedure as shown in Fig. 7. Signer feeds data to the hash function(SHA-512) and generates unique representation of data called hash of data. Hash value and signer private keys are used as 


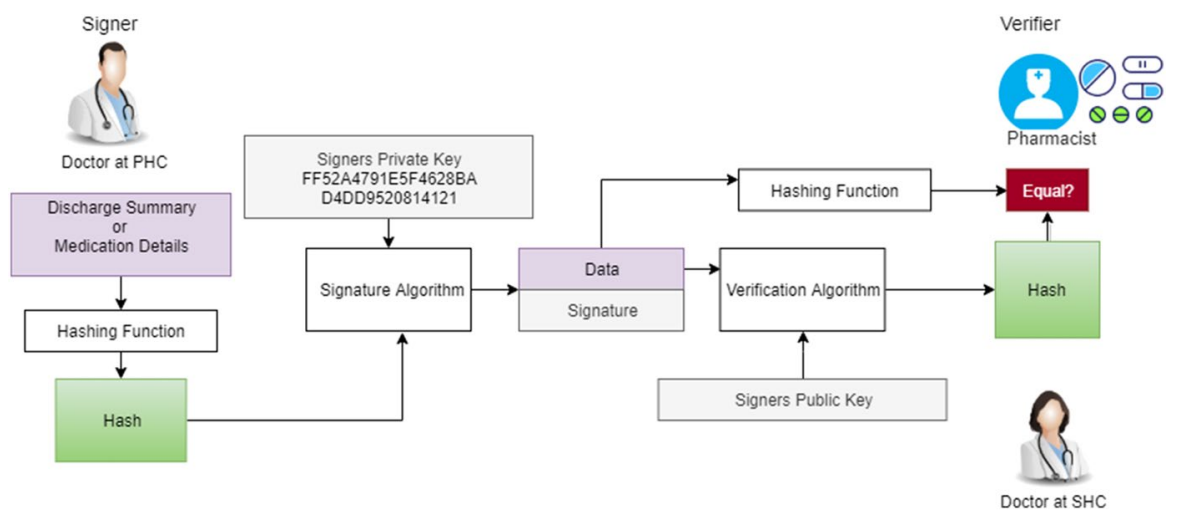

Fig. 7 Digitally Signed Prescription Process

an input to the signature algorithm called RSA which produces the digital signature on given hash. Both signature and the document are then sent to the verifier. Any other healthcare user from the other level can confirm the signature with the help of verification algorithm with signers public key.

At every level, the doctors authenticate the access using the digital signature. This feature enables and enhances the secure capturing and sharing of patient data as the

\section{PRESCRIPTION}

Secondary Hospital

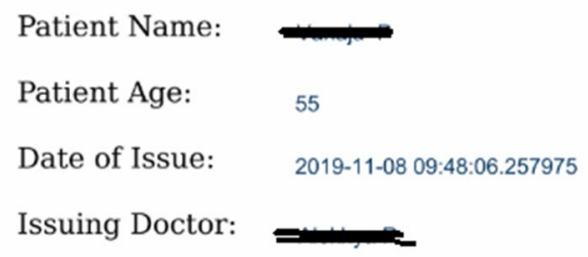

Medicine

Dosage

Nitroglycerin

$1-0-1$

\section{Aletlega $R$}

Doctor's Signature

$11 / 8 / 2019$ | $4: 57: 28$ PM IST

Fig. 8 Digitally Signed Prescription Sample 
EHR does not allow the doctors to approve any documents without the authorized digital signature as shown in Fig. 8.

In order to enable the doctors to digitally sign the documents, the Docusign REST-API is used which allows doctor to sign with few seconds. This enhanced the functioning of EHR in this model as not being able to approve documents with signature was a major drawback of previous traditional system. If doctor is creating digital signature for the first time he/she can select pre-drawn signature or choose to draw their own signature using mobile phone or graphic pad. Once the signature is ready, the doctor can digitally sign any medical document. For e.g after writing prescriptions, doctors received an email which redirected them to a page where they could review what they prescribed and then digitally approve it with their signature. This approved prescription was automatically visible to patients in real time in the form of printable PDF. Digitally signed documents can easily and securely be shared between providers, giving access to crucial health information when needed.

\subsection{Minimal or no configuration}

As this is a website it did not need any configuration in local machines as opposed to Desktop softwares. It was observed to be user-friendly. A chat bot was implemented in the stated model to help new users to get accustomed to the website which even guided/redirected them to requested pages. This bot was designed to respond to natural language in English text. To use chat bot healthcare users or patient needs to log into the website to perform all the activities. No threats about system crashing or security leaks were prevalent as it is web application with minimal setting user can access the application.

\subsection{Reduced cost}

The continual availability of patient records helped hospitals to avoid repetition of tests and reports. Also, the website had an eco-friendly facet as it curtailed paper usage and related costs. In a very visible sense, this work avoided paper usage and highly decreased its wastage. Instant availability of the patient health information makes healthcare professionals to spend more time on treatment rather than the writing patient details on paper based record.

\subsection{DICOM viewing option}

DICOM is an imaging information system which is used as an integral part of the EHR systems. The diagnostic image data obtained from the hospitals, clinics, imaging centers are globally stored in the cloud storage using DICOM standard format. The cloud computing provides storage space to archive the DICOM files and provide data security through security algorithm and SSL encrypted network. The centralized access allows healthcare professionals to access images from anywhere in the world for faster diagnosis decision. Patients can potentially obtain faster and more effective care when the DICOM Standard are available in healthcare enterprise network.

Doctors have the provision for viewing DICOM images with adequate clarity along with all the associated tags are shown in Fig. 9. Previous works were not designed to provide this option. Doctors usually had to use a separate software for viewing DICOM images which were generated by high-end lab technology like MRI machines. This 


\section{View DICOM of the Patient}

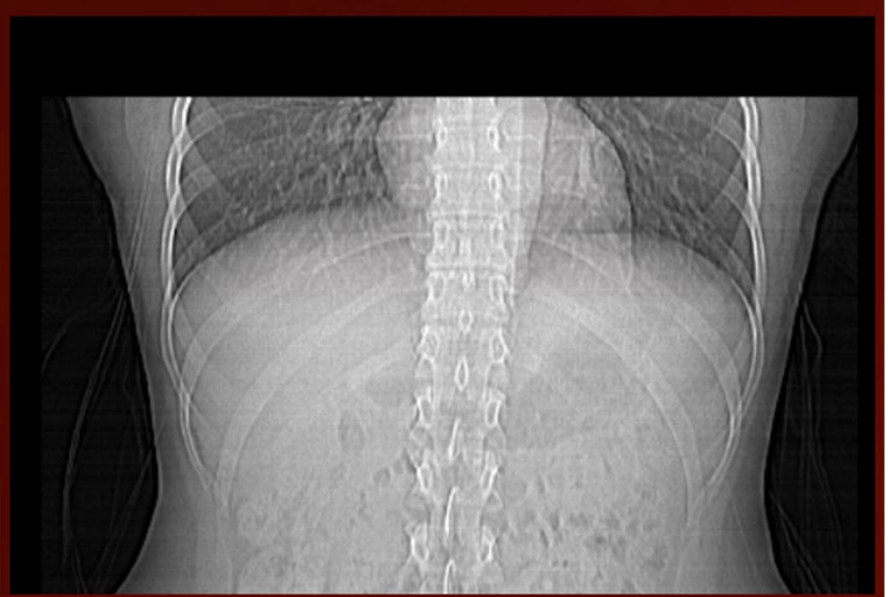

AcquisitionNumber : 1

BitsAllocated : 8

Bitsstored : 8

Columns : 512

ConversionType : WSD

HighBit : 7

ImageType : [ORIGINAL, 'SECONDARY, 'OTHER', 'ARC', 'DICOM', VALIDATION]

InstanceNumber : 1

Modality : OT

PatientID : 0

PatientName : Anonymized

Photometriclnterpretation : MONOCHROME2

PixelRepresentation : 0

Rows : 512

SOPClassUID : $1.2840 .10008 \cdot 5 \cdot 1.4 .1 .1 .7$

Fig. 9 DICOM View of the Patient

DICOM view model provides doctors with the option to view and analyze DICOM images online through their respective accounts. The DICOM viewer provides quick and easy access to medical image data. DICOM medical image format used in worldwide to store, exchange, and transmit and finally stored in cloud storage. The interface allows healthcare professionals to view the medical image in different dimension like $2 \mathrm{D}$ and $3 \mathrm{D}$ variation for better understanding of the image. 


\section{Conclusion}

Advancement in IT technology in healthcare sector has made it possible to maintain and manage the patient data in digital form in all levels of healthcare system. With the Government of India backing the Digital India movement, there have been a lot of advancements in the conglomerations of Healthcare and IT industry. This lead to the vast developments in the methods of record maintenance for the Indian healthcare sector. The objective of this research was to provide a simplified solution to completely support the healthcare service providers of PHC, SHC and THC with complete, accurate, adequate and timely patient information for providing quality care during the episode of care. It is a trump card in Healthcare domain as it provides secure real-time information retrieval. Now a days the web based EHR system is beneficial to store the patient's health information. This data should be globally available across various health care levels.

Various healthcare standards coding for recording and reporting such as ICD 10, SNOMED-CT, LOINC, DICOM and NDC has been incorporated. The system has better interoperability, maintainability, and scalability compared to traditional paper based health information system. The ultimate goal of this research is to completely support healthcare professionals of the different healthcare levels with required health information of the patient at all the time when it necessary.

The developed cloud based EHR system has been validated with real patient data of 60 from PHC and 40 form CHC. The test result indicated error-free capturing and sharing of patient data at various levels of the healthcare system. These features of EHR system would allow the planner and manager at all levels of the public health system to plan and conduct various preventive, curative and rehabilitative programs and ultimately support them in achieving national and international goals set for public healthcare.

Funding Open access funding provided by Manipal Academy of Higher Education, Manipal. No external funding has been received for this study.

\section{Compliance with Ethical Standards}

Conflict of interest Manohara Pai M.M declares that he has no confict of interest. Raghavendra Ganiga declares that he has no confict of interest. Radhika M Pai declares that she has no confict of interest. Rajesh Kumar Sinha declares that he has no confict of interest.

Ethical approval This article does not contain any studies with human participants or animals performed by any of the authors.

Open Access This article is licensed under a Creative Commons Attribution 4.0 International License, which permits use, sharing, adaptation, distribution and reproduction in any medium or format, as long as you give appropriate credit to the original author(s) and the source, provide a link to the Creative Commons licence, and indicate if changes were made. The images or other third party material in this article are included in the article's Creative Commons licence, unless indicated otherwise in a credit line to the material. If material is not included in the article's Creative Commons licence and your intended use is not permitted by statutory regulation or exceeds the permitted use, you will need to obtain permission directly from the copyright holder. To view a copy of this licence, visit http://creativecommons.org/licenses/by/4.0/. 


\section{References}

Affeldt, J.E.: The new quality assurance standard of the joint commission on accreditation of hospitals. West. J. Med. 132(2), 166 (1980)

Aocnp, D.: The evolution of the electronic health record. Clin. J. Oncol. Nurs. 19(2), 153 (2015)

Act, A.: Health insurance portability and accountability act of 1996. Publ. law 104, 191 (1996)

Bagchi, S.: Growth generates health care challenges in booming india. Can. Med. Assoc. J. 8, 981-983 (2008)

Balsari, S., Fortenko, A., Blaya, J.A., Gropper, A., Jayaram, M., Matthan, R., Sahasranam, R., Shankar, M., Sarbadhikari, S.N., Bierer, B.E., et al.: Reimagining health data exchange: an application programming interface-enabled roadmap for india. J. Med. Internet Res. 20(7), e10725 (2018)

Banff, Adoption and effective use of digital health across canada, https://www.infoway-inforoute.ca/ en/component/edocman/resources/i-infoway-i-corporate/annual-reports/2771-annual-report-20142015, (2015)

Basu, D.: The electronic health records system in the uk. https://www.centreforpublicimpact.org/casestudy/electronic-health-records-system-uk/, (2017)

Bahga, A., Madisetti, V.K.: A cloud-based approach for interoperable electronic health records (ehrs). IEEE J. Biomed. Health Inform. 17(5), 894-906 (2013)

Campbell, R.J.: Database design: what him professionals need to know. Perspectives in Health Information Management/AHIMA, American Health Information Management Association, vol. 1, (2004)

Chokshi, M., Patil, B., Khanna, R., Neogi, S.B., Sharma, J., Paul, V., Zodpey, S.: Health systems in india. J. Perinatol. 36(3), S9-S12 (2016)

J. Commission et al., The joint commission: over a century of quality and safety. https://www.joint commission.org/sitecore/media-library/deprecated-unorganized/imported-assets/tjc/system-folders/ assetmanager/tjc-history-timeline_through_20161pdf/, (2015)

Courtney, K.L., Shabestari, O., Kuo, A.: Enabling Health and Healthcare Through ICT: Available, Tailored and Closer, vol. 183. IOS Press, Amsterdam (2013)

Deodhar, N.: Primary health care in india. J. Publ. Health Policy 3(1), 76-99 (1982)

Dick, R.S., Steen, E.B., Detmer, D.E., et al.: The Computer-Based Patient Record: An Essential Technology for Health Care. National Academies Press, Washington (1997)

Digital Imaging and Communications in Medicine- DICOM. (2016). [Online]. Available: https://www. dicomstandard.org/

Ganiga, R., Pai, R.M., Sinha, R.K.: Security framework for cloud based electronic health record (ehr) system. Int. J. Electrical Comput. Eng. 10(1), 455 (2020)

Gopal, K.M.: Strategies for ensuring quality health care in india: experiences from the field. Indian J. Commun Med Off. Publ. Indian Assoc. Prev. Soc. Med. 44(1), 1 (2019)

Guo, Y., Kuo, M.-H., Sahama, T.: Cloud computing for healthcare research information sharing. In: 4th IEEE International Conference on Cloud Computing Technology and Science Proceedings. IEEE, (2012), pp. 889-894

Griebel, L., et al.: A scoping review of cloud computing in healthcare. BMC Med. Inform. Decis. Mak. 15(1), 17 (2015)

Ganiga, R., Pai, M.R., Pai, M., Sinha, R.K.: Cloud enabled standard electronic health record architecture for indian healthcare sector. Indian J. Publ. Health Res. Dev. 8(4), 554-562 (2017)

Ganiga, R., Pai, R.M., Pai, M.M., Sinha, R.K., et al.: A preliminary study of real-time capturing and sharing of routine health data among the public health professionals. Indian J. Community Med. 45(2), 176 (2020)

Gkoulalas-Divanis, A., Loukides, G., Sun, J.: Publishing data from electronic health records while preserving privacy: A survey of algorithms. J. Biomed. Inform. 50, 4-19 (2014)

Giaedi, T.: The impact of electronic medical records on improvement of health care delivery. Libyan J. Med. 3(1), 4 (2008)

Gold, M., McLAUGHLIN, C.: Assessing hitech implementation and lessons: 5 years later. Milbank Q. 94(3), 654-687 (2016)

Hersh, W.R.: The electronic medical record: promises and problems. J. Am. Soc. Inform. Sci. 46(10), 772-776 (1995)

Health Level 7 (HL7) - International. (2016). [Online]. Available: https://www.hl7.org/

Jaroudi, S., Payne, J.D.: Remembering lawrence weed: a pioneer of the soap note. Acad. Med. 94(1), 11 (2019)

Kruse, C.S., Stein, A., Thomas, H., Kaur, H.: The use of electronic health records to support population health: a systematic review of the literature. J. Med. Syst. 42(11), 214 (2018) 
Lærum, H., Karlsen, T.H., Faxvaag, A.: Effects of scanning and eliminating paper-based medical records on hospital physicians' clinical work practice. J. Am. Med. Inform. Assoc. 10(6), 588-595 (2003)

National Health Portal, Ministry of Health and Family Welfare, Government of India, EHR Standards. (2016). [Online]. Available: https://www.nhp.gov.in/NHPfiles/EHR-Standards-2016-MoHFW.pdf

SNOMED International, SNOMED CT Basics. (2016). [Online]. Available: https://www.snomed.org/ resources/resources

Logical Observation Identifiers Names and Codes(LOINC): Origins of LOINC. (2016). [Online]. Available: https://loinc.org/about/

RxNorm: Unified Medical Language System. (2016). [Online]. Available: https://www.nlm.nih.gov/ research/umls/rxnorm/index.html

Ministry of Health and Family Welfare, Government of India, Draft National Digital Health Blueprint. (2019). [Online]. Available: https://main.mohfw.gov.in/sites/default/files/Final\%20NDHB\%20rep ort_0.pdf

Ministry of health and family welfare, Electronic health record standards for india. (2013). [Online]. Available: https://www.nhp.gov.in/NHPfiles/ehr_2013.pdf

N. H. I. Technology, Electronic health record (ehr) system testing plan. https://www.healthit.gov/sites/defau 1t/files/playbook/pdf/ehr-system-testing-plan.pdf, (2016)

Outlook, G.H.: Global health care sector issues in 2019. https:/www2.deloitte.com/content/dam/Deloitte/ global/Documents/Life-Sciences-Health-Care/gx-lshc-hc-outlook-2019.pdf, (2019)

D. P. A. of India, Personal data protection bill 2019, (2019). [Online]. Available: https://www.businesstoday. in/current/policy/personal-data-protection-bill-2019-central-government-power/-may-undermine-priva cy-of-citizens-people/story/392186.html

P. O. of Science and Technology, Electronic health records. https://researchbriefings.parliament.uk/Resea rchBriefing/Summary/POST-PN-0519, (2016)

Lehr, B.: Germany: Healthcare system and ehealth strategy. http://www.ehealthforregions.net/about-us/ partners/germany.html, (2016)

Lee, M., Heo, E., Lim, H., Lee, J.Y., Weon, S., Chae, H., Hwang, H., Yoo, S.: Developing a common health information exchange platform to implement a nationwide health information network in south korea. Healthc. Inform. Res. 21(1), 21-29 (2015)

J. C. International: Apollo hospital:it excellence. https://www.apollohospitals.com/patient-care/clinicalquality-and-outcomes/it-excellence, (2019)

M. R. Foundation: Sankara nethralaya. https://www.sankaranethralaya.org/patient-care-eye-q.html, (2019)

Rozenblum, R., Jang, Y., Zimlichman, E., Salzberg, C., Tamblyn, M., Buckeridge, D., Forster, A., Bates, D.W., Tamblyn, R.: A qualitative study of canada's experience with the implementation of electronic health information technology. Cmaj 183(5), E281-E288 (2011)

Rehmann, W.: E-health law in germany. https://www.lexology.com/library/detail.aspx?g=83f3f929-7be74383-a66b-ed1dedeb912d, (2016)

Sharma, N.C.: Adoption of e-medical records facing infra hurdles. https://www.livemint.com/Politics/ CucBmKaoWLZuSf1 Y9VaafM/Adoption-of-emedical-records-facing-infra-hurdles-Report.html, (2018)

Sarbadhikari, S.N.: Will health informatics gain its rightful place for ushering in digital india? Indian J. Community Med. Off. Publ. Indian Assoc. Prev. Soc. Med. 43(2), 126 (2018)

Sayles, N.B., Gordon, L.L.: Health information management technology: An applied approach. 5th Edition, Am. Health Inform. Manag. Assoc Press, (2013)

Sarbadhikari, S.N.: The role of standards for digital health and health information management. J. Basic Clin. Res. (JBCR) 6(1), 1 (2019)

Soi, A.: Max healthcare. https://www.maxhealthcare.in/hospitals-in-india, (2019)

Stausberg, J., Koch, D., Ingenerf, J., Betzler, M.: Comparing paper-based with electronic patient records: lessons learned during a study on diagnosis and procedure codes. J. Am. Med. Inform. Assoc. 10(5), 470-477 (2003)

Sweeney, J.: Healthcare informatics. On-Line Journal of Nursing Informatics 21(1), 1-4 (2017)

Srivastava, S.K.: Adoption of electronic health records: a roadmap for india. Healthc. Inform. Res. 22(4), 261-269 (2016)

Sarbadhikari, S.N., et al.: Digital health in india-as envisaged by the national health policy (2017). BLDE Univ. J. Health Sci. 4(1), 1 (2019)

Takeda, H.: What has changed after dr. lawrence weed's paper in 1968? Yearbook of Med. Inform. 8(01), 218-220 (1999)

World Health Organization, International Classification of Diseases- Version 11 (ICD-11). (2016). [Online]. Available: https://www.who.int/classifications/icd/en/ 
Warren, J.J., Matney, S.A., Foster, E.D., Auld, V.A., Roy, S.L.: Towards interoperability: a new resource to support nursing terminology standards. Comput. Inform. Nurs. CIN 33(12), 515 (2015)

Wright Jr., J.R.: The american college of surgeons, minimum standards for hospitals, and the provision of high-quality laboratory services. Arch. Pathol. Lab. Med. 141(5), 704-717 (2017)

Publisher's Note Springer Nature remains neutral with regard to jurisdictional claims in published maps and institutional affiliations. 